\title{
The postdoctoral apprenticeship
}

\author{
Much has been written already about whether the scientific machine is \\ churning out too many PhDs and postdocs when there are a limited number \\ of academic jobs and the competition for funding and space in competitive \\ journals is intense. But gratifyingly, there exists a vast array of other \\ scientific careers. We need to mentor and advise trainees about the diverse \\ and rewarding professional opportunities that are available beyond the \\ postdoctoral apprenticeship period.
}

Given that many trainees do opt for a modicum of postdoctoral training, what is the right pay scale for them, and why, until now, have we paid them so pitifully? There is a historical precedent that up until now, most universities have followed - and the time is ripe to reevaluate. The individuals with the determination and grit to get graduate degrees are currently offered an entry-level salary (at many institutions that follow the NIH guidelines) that comes out to between \$11 (80h/week) and \$21 (40h/ week) an hour ( $\$ 44,000$ salary). These rates don't come anywhere near the same range as skilled, entry-level positions in fields outside of science. Beyond my JCI role, I now also work at Memorial Sloan Kettering Cancer Center (MSK), and can report that we have joined several peer institutions in raising postdoc pay to a minimum of $\$ 50,000$ a year. This new minimum salary is in compliance with the changes to the Fair Labor Standards Act, which mandates that after December 1, 2016, employers must pay overtime to any salaried employee earning less than $\$ 47,476$. [There is some ongoing debate as to whether postdocs are employees, but why quibble?] I can think of few postdocs at MSK, nor many at any of the five other universities I've been affiliated with, who work fewer than 40 hours a week. We have been lucky at MSK to consistently recruit super high-quality postdocs and are making this move to a $\$ 50,000$ baseline salary to ensure that we continue to attract and retain talented postdocs. It is our hope to continue to increase this minimum, keeping in mind that the cost of living in NYC is higher, but all the while keeping in mind the tight budgets imposed by many funding agencies in the USA. Regardless of these constraints, all scientific institutions should be encouraged to meet or exceed this new threshold.

It is an enormously exciting time to be a scientist today. We should be proud to be scientists, and to be training future scientists no matter what venue they choose.

\section{Ushma S. Neill Editor at Large}

